\title{
Cardinal Signs of Autism
}

\section{Grace Lalana Christopher*}

Grace Specialist Clinic, India

One can ask what Autism is, it is a neuro-developmental disorder best described by the terminology Autism Spectrum Disorder (ASD) which encompasses the previous diagnoses of autism, Asperger syndrome, pervasive developmental disorder not otherwise specified (PDD-NOS) and childhood disintegrative disorder. The most noticeable characteristic is a triad of disturbances in social interaction and communication in multiple contexts together with restricted interests and repetitive behaviour patterns. The diagnosis is exclusively based on symptoms and the presenting profile. As Autism develops before three years and diagnosable by one and a half years of age, autistic children can be identified early, though often it may be associated with other developmental disorder of brain function including mental retardation, cerebral palsy, learning disability and attention deficit disorder with poor language and social interactions.

The lack of social interaction and communication skills with poor eye contact, gestures and facial expression is demonstrated by lack of joint attention. Normally the desire for an object is indicated by the child, pointing or verbalizing usually to the mother is known as joint attention which normally emerges around nine months of age and is established by eighteen months of age. This is also referred to as 'triadic exchanges' which is a sophisticated form of communication of internal emotional states and intent between two individuals in reference to a third party or object by using eye contact, gaze, gesture or touch.

Social referencing is also very important which is characterized by a child looking at the parent to see if the adult is watching them and what they are doing or to check to see if what they are doing is okay or safe. This skill plays a critical role in social and language development and initiating of joint attention by the child shows that the child is socially motivated [1]

The first form of joint attention of expressing emotion or drawing attention to an object etc. is known as declarative or proto declarative behaviour such as a child pointing to an object and glancing between the object and mother while smiling. While the second type of joint attention is referred to as imperative or proto imperative such as the child guiding parent's hand to reach a toy which is out of reach also requesting the adult to give the toy to the child. Thus both declarative and imperative joint attention behaviours are impaired in a child with autism as shown in Figure 1.

Parents generally observe these signs of Autism in first two to three

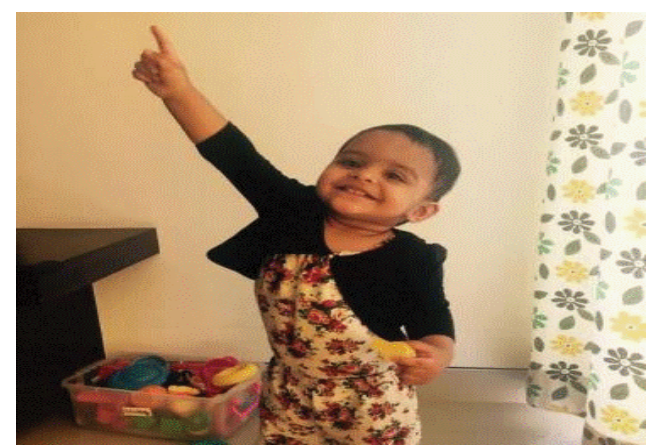

Figure 1: Joint attention or 'Protodelerative' behaviour lacking in a child with autism. years of their child's life as these signs tend to develop gradually, while some parents report that their child reached developmental milestone at a normal pace and then regressed. Autism is estimated to affect around one per cent of all children with marked speech, language and communication needs. Autistic child is often withdrawn and spends hours in solitary play. They have poor eye contact or orientation to one's name even when called by name with lack reciprocal smile.

Language is poor and they rely on nonverbal communication. If speech is present it is usually repetition of words or jargon. They have narrow range of interest or sameness lacking imagination; also they do not engage in pretend play which starts before the age of two years in normal children. The child tries to maintain a routine and throws tantrum like rage if the routine is disrupted.

Pointers towards autism in a child:

Absence of babbling or use of gestures by 1 year.

Absence of speaking any single word by 16 months.

Absence of use of purposeful two word phrases by 2 years.

Delay in appearance of social smile.

Delay in the appearance of stranger anxiety.

Any loss of language or social skills at any age.

Absence of joint attention or as 'triadic exchanges' social referencing, proto declarative behaviour as well as or proto imperative behaviour.

An early diagnosis is crucial as early intervention based on communication and behavioural principles will result in improvement of presenting clinical features, even though medications may be used to try and improve certain associated problems it is not curative.

\section{Reference}

1. Christopher GL (2018) Setting and implementing effective boundaries, Second Edition, 174.
*Corresponding author: Christopher GL, Grace Specialist Clinic, India, Tel: +9199728229403; E-mail: drgrace80@yahoo.com

Received May 10, 2018; Accepted May 28, 2018; Published June 03, 2018

Citation: Christophe GL (2018) Cardinal Signs of Autism. Mol Biol 7: 212. doi: 10.4172/2168-9547.1000212

Copyright: $\odot 2018$ Christophe GL. This is an open-access article distributed under the terms of the Creative Commons Attribution License, which permits unrestricted use, distribution, and reproduction in any medium, provided the original author and source are credited. 